\title{
VLC Quantum Fusion
}

\author{
$1^{\text {st }}$ George Suciu \\ $R \& D$ Department \\ BEIA Consult International \& UPB \\ Bucharest, Romania \\ george@beia.ro \\ $4^{\text {th }}$ Alexandru Drosu \\ $R \& D$ Department \\ BEIA Consult International \\ Bucharest, Romania \\ alex.drosu@beia.ro
}

\author{
$2^{\text {nd }}$ Andrei Scheianu \\ $R \& D$ Department \\ BEIA Consult International \\ Bucharest, Romania \\ andrei.scheianu@beia.ro \\ $5^{\text {th }}$ Roberta Darabană \\ $R \& D$ Department \\ BEIA Consult International \\ Bucharest, Romania \\ roberta.darabana@beia.ro
}

\author{
$3^{\text {rd }}$ Ioana Petre \\ R\&D Department \\ BEIA Consult International \\ Bucharest, Romania \\ ioana.petre@beia.ro
}

\begin{abstract}
Visible Light Communication (VLC) is an innovative and proficient wireless communication technology that permits LED lighting and optical communications to work together by modulating the LED light source. For security in the Internet of Things (IoT), VLC communications face different challenges that were disputed in various research papers. VLC communication incorporated with the rising innovation of the IoT initiates a wide range of indoor applications. Quantum technology can also be integrated in VLC. Quantum dots (QDs) are promising materials regarding lighting and display because of their easy customizable emission wavelength, restricted Full Width at Half Maximum (FWHM) and superior quantum performance. This paper represents the state-of-the-art in the field of VLC Quantum. It presents a study with the most noticeable research activities and experimental results related to the VLC Quantum technology. Also, we propose a Q-IoT platform prototype which represents an innovative way to improve the IoT security and to create a safer network environment to operate within.
\end{abstract}

Keywords-Quantum Theory, Quantum Dots, Visible Light Communication, WLED, LED

\section{INTRODUCTION}

CQDS semiconductors (Colloidal Semiconductor Quantum Dots) represents nanoparticles that present a high light-emitting efficiency. This technology makes the main focus of the research activities undertaken in the academic or industrial domain during the last 20 years [1]. While this kind of semiconductors can be used as fluorescent tags (for bio-assays and bio-imaging [2]), as photovoltaic elements [3] or as laser-gain materials and LEDs (Light-Emitting Diodes) [4]-[7], the most noticeable utility of them was remarqued in the case of hybrid light-emitting displays or devices, under the form of phosphors [8-10]. Due to their high PLQY (Photoluminescence Quantum Yield), solution processability, narrow emission linewidths and broad absorption, the CQDs semiconductors represents a good alternative for the rare-earth phosphor element which can be found in the worldwide LED sources existent on the market. Also, the CQDs semiconductors can be integrated in systems designed for the establishment of data communication links.

The CQDs semiconductors can be used in the process of color-converting of the violet/blue micro-sized LED sources (InGaN $\mu$ LEDs) [11]-[12]. These light emitting sources presents transmitting areas comprised between $10 \mu \mathrm{m}^{2}$ and $10^{3} \mu \mathrm{m}^{2}$. Also, the InGaN LEDs can be distributed in array formats which can contain thousands of pixels per square $\mathrm{mm}$ and present the capability of integration with CMOS electronics in case of temporal and spatial control. The result of such an operation will be a micro display technology available for integration in several applications [13]-[15]. Regarding the efficiency of the InGaN LEDs emission, the wavelength region with the best efficiency is the blue/violet spectrum [16]. The CQDs semiconductors provides a better solution of processability, giving an increased flexibility in the fabrication process of different hybrid devices. In terms of the VLC (Visible Light Communication) technology, the $\mu L E D$ presents potential because of the available spatial multiplexing. Also, another important characteristic of the $\mu L E D$ s related to the VLC field is the high-speed modulation [15]-[17], several VLC communication links with multi$\mathrm{GB} / \mathrm{s}$ speeds being demonstrated in [18].

The Visible Light Communication and the LiFi extension [19] represent a new communication technology that gained a lot of popularity during the last years be-cause of the specific advantages related to the spectrum of frequencies that can be used to establish communication channels [19][21]. The InGaN LEDs efficiency and rapid development process meet the market requirements regarding the need of an industry based on solid-state lighting [20]. The VLC technology has the potential to transform the traditional lighting sources in specific components of a new wireless communications network capable to handle the enormous amount of data specific to mobile applications and IoT (Internet of Things) [20].

As any other new technology several challenges exist. One of them is the fact that the InGaN LED sources doesn't provide the possibility of modulation at high data speeds in order to obtain efficient communication channels, due to the fact that the specific bandwidth of phosphor LEDs emitting white light is lower than $2 \mathrm{MHz}$ [21]. Therefore, the light converters that present smaller PLs (Photoluminance Lifetimes) are required [12]. Furthermore, the limited linewidth emission of the CQDs represents an important parameter in order to maintain a high degree of light quality and color purity.

The article has the following structure: in Section II is presented a comprehensive study regarding the most notable research activities related to the VLC Quantum Technology, a hybrid LED system based on CQD color converter for VLC communications establishment is presented in Section III, Section IV presents a Q-IoT prototype designed for VLC Quantum Technology while Section V contains the conclusions of the paper and the future work.

\section{RELATED WORK}

In [18] the authors explore the field of high transfer rate VLC using Gallium Nitride LEDs (based on Quantum efficiencies). It is stated that the main body of the paper is 
focused on researching the technologies that can offer multi Git/s transfer rates in VLC systems while also using the Quantum concept. After that, Gallium Nitride-based LEDs are fully described. The authors state that in order to obtain a regime where the internal quantum efficiency remains relatively high an increased area that will allow at a higher current than its operating range may be needed.

Paper [22] illustrates a system based on NRZ-OOK type of modulation which uses a commercial phosphorescent WLED (White Light LED), an analog pre-equalizer and post-equalizer in order to obtain a $550 \mathrm{Mbit} / \mathrm{s}$ real-time VLC link. In this way, a high-speed and low complexity VLC system was acquired.

Another interesting study was elaborated in 2010, by McKendy et al [23]. The above-mentioned paper reveals the E-O (Electrical-to-Optical) modulation band-widths estimated from single pixels of GaN, based of uLED clusters at the peak wavelengths: 370,405 and $450 \mathrm{~nm}$. The distance between the pixels was around $72 \mu \mathrm{m}$. As a result, the maximum bandwidth was $245 \mathrm{MHz}$, at $450 \mathrm{~nm}$ distance between the uLEDs.

Superior modulation bandwidths from uLEDS have been published in various re-searches. For example, paper [24] proposed a blue emitting uLED with a gallium-doped zinc oxide current-confining aperture, which permitted a modulation band-width of $225 \mathrm{MHz}$. In [25] is presented a 14-element uLED array with a modulation bandwidth higher than $450 \mathrm{MHz}$, whereas paper [26] reports an approximative bandwidth of $400 \mathrm{MHz}$ at a peak emission of $500 \mathrm{~nm}$.

The above-mentioned experimental results demonstrate that very high modulation bandwidths can be obtained by using uLED structures, in comparison with the conventional chip LEDs.

In [27] multiple quantum well diodes suitable for inplane VLC are presented. The particularity of this approach is that multiple quantum well diodes can be used as both receivers and transmitters in an in-line VLC system. The authors also include an experimental demonstration for the VLC with diodes capable of simultaneous emission and photodetection.

The proposed diodes use an InGaN/GaN p-n junction with multiple quantum wells. For the actual implementation of the VLC system, a GaN silicon wafer was used along with multiple other layers with different widths ( $\mathrm{nm}$ range). The resulting system is very compact and uses suspended compact waveguides as well. It is mentioned that the waveguide's performance is evaluated using the method of finite difference time domain and that point-like source with a wavelength of $450 \mathrm{~nm}$ is used for the simulation.

For the actual implementation of the VLC system, more than 90000 positions in 100 independent trials were used. The results are fully explained, with scenario description and graphical results for the errors.

\section{EXAMPLE OF HYBRID LED SYSTEM BASED ON CQD COLOR CONVERTER FOR VLC COMMUNICATIONS ESTABLISHMENT}

Given the fact that CQD started to be used intensively in the manufacturing process of different lighting devices and displays, in this subchapter will be presented an example of a result obtained by combining CQDs with GaN electroluminescent diodes of small sizes, in order to develop optical sources capable of supporting the establishment of VLC communication channels.

The hybrid LED sources used in this situation consist of CQD color converters that presents in their frame a flexible glass structure, which take directly physical contact with a polished sapphire substrate specific to an $\mu \mathrm{LED}$ light emitting area with a wavelength of $450 \mathrm{~nm}$. From the point of view of the specifications of the $\mu$ LEDs that compose the lighting area, each $\mu$ LED has a square shape and an emission area of $100 \mu \mathrm{m} \times 100 \mu \mathrm{m}$. The CdSxSe1-x/ZnS alloyed-core CQDs emitting red $(630 \mathrm{~nm}$ wavelength), orange $(575 \mathrm{~nm}$ wavelength) and green light (540 nm intrinsic wave-length) are used as color conversion material. The nominal diameter of the CQD components is $6 \mathrm{~nm}$ and their specific emission wavelength is determined by the composition of the alloy used. In order to obtain a CQD film it is necessary to dissolve the colloidal quantum dots in chloroform at different concentrations, as well as storing them on a thin and flexible glass membrane with a thickness of $30 \mu \mathrm{m}$. The obtained CQD film requires air drying for a period of several minutes, after which a glass membrane identical to the one specified above will be placed on top of it. The edges of the obtained film are being sealed through an ultra-violet curable epoxy (NOA65). The last stage of this process consists in illuminating the obtained sample with UV light with a luminous intensity per $\mathrm{cm}^{2}$ of $100 \mathrm{~mJ}$. The motivation behind the choice of the flexible glass was that it represents a quality oxygen barrier that has also been used in the encapsulation of laser-based solutions. The increased degree of flexibility facilitates the process of integration of the $\mu$ LEDs with the color converters.

The spectrum of the color-converted $\mu$ LED specific to each wavelength mentioned above can be seen in Fig. 1 (the spectral density response characteristic to the optical detector is calibrated, while the intensity data is normalized to the maximum green light emission threshold). The peak values recorded for the specific emissions of the three wavelengths were $650 \mathrm{~nm}$ for red devices, $606 \mathrm{~nm}$ for orange devices and $572 \mathrm{~nm}$ for green devices.

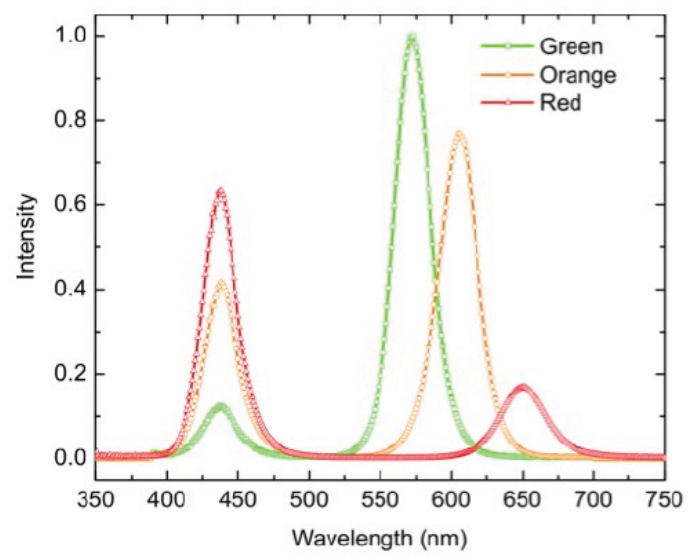

Fig. 1. The spectrum of the hybrid color-converted $\mu$ LEDs [28]

Fig. 2 illustrates the time settled light emissions measurements on the samples. The light emitted by every one of the three CQD color shading converters rots decrease with one nanosecond per timescale. The orange and green CQD films PL decreases are mono-exponential (over the 
considered range of $0-80 \mathrm{~ns}$ ), presenting comparative lifetimes of $33.5+/-0.5 \mathrm{~ns}$, and, $34+/-0.5 \mathrm{~ns}$.

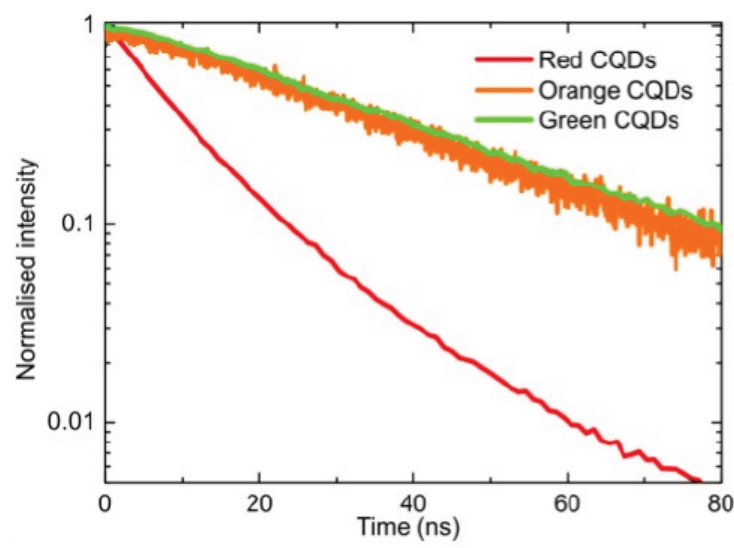

Fig. 2. The luminescence lifetime of the green, orange and red CQDs [28]

Once the optical properties specific to the CQD color converters integrated in $\mu$ LEDs have been established, the establishment of VLC (Visible Light Communication) channels based on hybrid $\mu$ LED color converters can be achieved through the usage of the OFDM (Orthogonal Frequency Division Multiplexing) modulation technique as the main data encoding method.

An example of an $\mu$ LED device having integrated color converters located on a visible light communication link demonstrator can be analysed in Fig.3. More details related to this experiment can be found in [28].

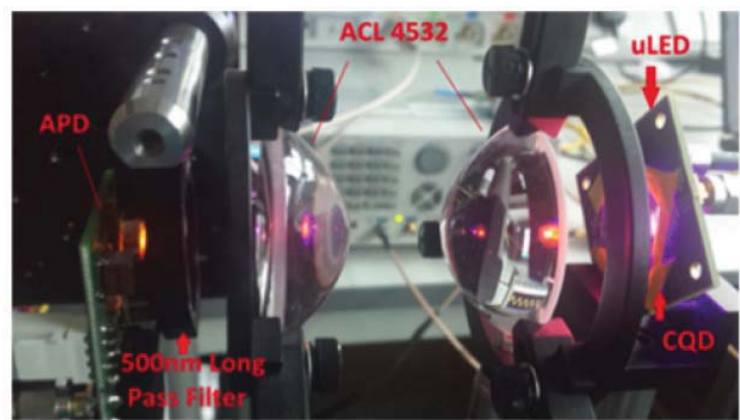

Fig. 3. Example of an $\mu$ LED device having integrated color converters located on a VLC link demonstrator [28]

\section{Q-IOT PROTOTYPE FOR VLC QUANTUM TECHNOLOGY}

The work presented in [29] was elaborated with IoT devices. The VLC system uses Arduino UNO boards as processing units for both the receiver and the transmitter (including also data acquisition). The work was later extended by replacing the microcontroller-like processing units with more capable Raspberry Pi mini computers. This change brought multiple advantages over the old approach, as the Raspberry Pi features a fully functional Linux-based operating systems. Also, the boards integrate Wi-Fi and Bluetooth chips, which can provide full integration of the VLC system with the existing communication protocols. This type of integration can also provide the VLC system access to Cloud or virtualization environments.

However, by using more potent IoT devices connected through multiple communication protocols, the most critical issue of the IoT concept is highlighted: security.

It is estimated that by 2020 , the number of Internet- connected devices will exceed 50 billion - with a significant percent of those devices being use-orientated IoT devices. This category includes smart household items, alarms, sensors - devices with limited processing capabilities and not security orientated, but with time-critical requirements [30].

No matter which type of IoT device would be integrated into a VLC system, the whole concept could benefit from the advantages of Quantum Computing. The critical issue with this integration is the imminent threat to the standard security paradigm due to enhanced computational problems which add to the existing security issues in the IoT world.

Considering the experiment presented in the previous section, BEIA Q-IoT prototype has the purpose to resolve the issues in the IoT quantum technology. This platform could present the potential of creating a safer network environment to operate in, by using a quantum key distribution and a quantum random number generator.

An integrated VLC system would benefit from an IoT quantum-safe technology, creating an extra-secure interface for connections between fog/edge and cloud. For this, a Raspberry Pi-based prototype was developed. The prototype can be observed in Fig.4. Currently, this prototype is in the optimization stage, following its performance testing once this process will be completed.

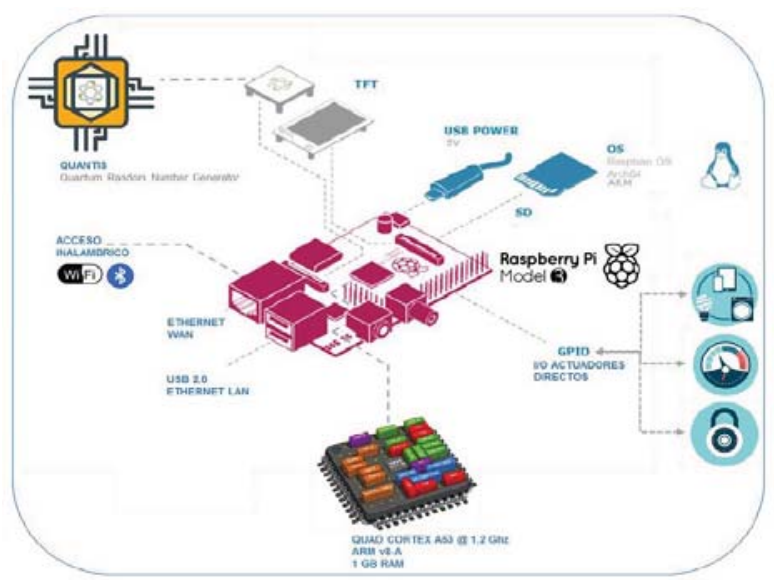

Fig. 4. BEIA Q-IoT prototype

\section{CONCLUSIONS}

In this article a State-of-The-Art presentation was realised regarding the most notable research activities undertaken in the direction of VLC quantum technology, as well as an example of a Q-IoT platform that was created as a solution for the quantum IoT Technology specific problems. As future work, the interconnection between the Q-IoT platform and blockchain technology will be tried to address different data packets.

\section{ACKNOWLEDGMENT}

This paper has been supported in part by UEFISCDI Romania under grant no. 97/2017, Hybrid VLC/IR-RF Communication for Smart Space Based on Multi-Functional Thermal Image Sensor Module (VLC/IR-RF) and funded in part by European Union's Horizon 2020 research and innovation program under grant agreement No. 777996 (SealedGRID project) and No. 787002 (SAFECARE project). 


\section{REFERENCES}

[1] J. Y. Kim, O. Voznyy, D. Zhitomirsky, and E. H. Sargent, "25th Anniversary Article: Colloidal Quantum Dot Materials and Devices: A Quarter-Century of Advances", Adv. Mater., vol. 25, pp. 49865010,2013

[2] I. L. Medintz, H. T. Uyeda, E. R. Goldman, and H. Mattoussi, "Quantum dot bioconjugates for imaging, labelling and sensing," Nat. Mater., vol. 4, no. 6, pp. 435-446, Jun. 2005.

[3] D. Vasudevan, R. R. Gaddam, A. Trinchi, and I. Cole, "Core-shell quantum dots: Properties and applications," Journal of Alloys and Compounds, vol. 635, pp. 395-404, 2015.

[4] T.-H. Kim et al., "Full-colour quantum dot displays fabricated by transfer printing," Nature Photonics, vol. 5, pp. 176-182, 2011.

[5] V.I. Klimov, A. A. Mikhailovsky, Su Xu, A. V. Malko, J. A. Hollingsworth, C. A. Leatherdale, H.-J. Eisler and M. G. Bawendi, "Optical gain and stimulated emission in nanocrystal quantum dots," Science, vol. 290, no. 5490, p. 314, 2000.

[6] C. Dang, J. Lee, C. Breen, J. S. Steckel, S. Coe-Sullivan, and A. Nurmikko, "Red, green and blue lasing enabled by single-exciton gain in colloidal quantum dot films," Nat. Nanotechnol., vol. 7, no. 5, pp. 335-339, 2012.

[7] B. Guilhabert, C. Foucher, A.-M. Haughey, E. Mutlugun, Y. Gao, J. Herrnsdorf, H. D. Sun, H. V. Demir, M. D. Dawson, and N. Laurand, "Nanosecond colloidal quantum dot lasers for sensing," Optics Express, vol. 22, no. 6, 7308-7319, 2014.

[8] T. Erdem and H. V. Demir, "Colloidal nanocrystals for quality lighting and displays: milestones and recent developments," Nanophotonics, vol. 5, no. 1, pp. 74-95, 2016.

[9] S. Nizamoglu, G. Zengin, and H. V. Demir, "Color-converting combinations of nanocrystal emitters for warm-white light generation with high color rendering index," Appl. Phys. Lett., vol. 92, 031102, 2008.

[10] H. V. Demir, S. Nizamoglu, T. Erdem, E. Mutlugun, N. Gaponik, and A. Eychmüller, "Quantum dot integrated LEDs using photonic and excitonic color conversion," Nano Today, vol. 6, no. 6, pp. 632-647, 2011.

[11] B. Guilhabert, D. Elfstrom, A. J. C. Kuehne, D. Massoubre, H. X. Zhang, S. R. Jin, A. R. Mackintosh, E. Gu, R. A. Pethrick, and M. D. Dawson, "Integration by self-aligned writing of nanocrystal/epoxy composites on InGaN micro-pixelated light-emitting diodes," Optics Express, vol. 16, no. 23, pp. 18933-18941, 2008.

[12] N. Laurand, B. Guilhabert, J. McKendry, A. E. Kelly, B. Rae, D. Massoubre, Z. Gong, E. Gu, R. Henderson, and M. D. Dawson, "Colloidal quantum dot nanocomposites for visible wavelength conversion of modulated optical signals," Optical Materials Express, vol. 2, no. 3, pp. 250-260, 2012.

[13] H. X. Zhang, D. Massoubre, J. McKendry, Z. Gong, B. Guilhabert, C. Griffin, E. Gu, P. E. Jessop, J. M. Girkin, and M. D. Dawson, "Individually-addressable flip-chip AlInGaN micropixelated light emitting diode arrays with high continuous and nanosecond output power," Optics Express, vol. 16, no. 13, pp. 9918-9926, 2008.

[14] J. Herrnsdorf, Y. Wang, J. J. D. McKendry, Z. Gong, D. Massoubre, B. Guilhabert, G. Tsiminis, G. A. Turnbull, I. D. W. Samuel, N. Laurand, E. Gu and M. D. Dawson, "Micro-LED pumped polymer laser: A discussion of future pump sources for organic lasers," Laser \& Photonics Review, vol. 7, pp. 1065-1078, 2013.

[15] J. McKendry, R. P. Green, A. E. Kelly, Z. Gong, B. Guilhabert, D. Massoubre, E. Gu, and M. D. Dawson, "High Speed Visible Light Communications Using Individual Pixels in a Micro Light-Emitting Diode Array," IEEE Photon. Technol. Lett., vol. 22, no. 18, pp. 13461348,2010 .
[16] M. R. Krames, O. B. Shchekin, R. Müller-Mach, G. O. Müller, L. Zhou, G. Harbers, and M. G. Craford, "Status and future of highpower lightemitting diodes for solid-state lighting," J. Disp. Technol., vol. 3., no. 2, pp. 160-175, 2007.

[17] R. X. Ferreira et al., "High bandwidth GaN-based micro-LEDs for multi-Gb/s visible light communications," IEEE Photonics Technology Letters, vol. 28, no. 19, 2023-2016, 2013.

[18] S. Rajbhandari, J. J. D. McKendry, J. Herrnsdorf, H. Chun, G Faulkner, H. Haas, I. M. Watson, D. O'Brien, and M. D. Dawson, “A review of gallium nitride LEDs for multi-gigabit-per-second visible light data communications," Semicond. Sci. Technol., vol. 32, 023001, 2017

[19] H. Haas, L. Yin, Y. Wang, and C. Chen, "What is Lifi?," Journal of Lightwave Technology, vol. 34, no. 6, pp. 1533-1544, 2015.

[20] H. Haas, "A light-connected world," Physics World, vol. 29, no. 8, pp. 30-34, 2016.

[21] D. C. O’Brien, L. Zeng, H. Le-Minh, G. Faulkner, J. W. Walewski, and S. Randel, "Visible light communications: Challenges and possibilities," in IEEE 19th Int. Symp. Pers. Indoor Mob. Radio Commun., pp. 1-5, 2008.

[22] H. Li, X. Chen, J.Guo, H. Chen, A $550 \mathrm{Mbit} / \mathrm{s}$ real-time visible light communication system based on phosphorescent white light LED for practical high-speed low-complexity application, Optics Express (22), 2014, DOI:10.1364/OE.22.027203

[23] J. J. D. McKendry, R. P. Green, A. E. Kelly, G. Zheng, B. Guilhabert, D. Massoubre, E. Gu, and M. D. Dawson, "High-speed visible light communications using individual pixels in a micro light-emitting diode array," IEEE Photonics Technol. Lett., vol. 22, no. 18, pp. 1346-1348, 2010.

[24] C. L. Liao, Y. F. Chang, C. L. Ho, and M. C. Wu, "High-speed GaNbased blue light-emitting diodes with gallium-doped $\mathrm{ZnO}$ current spreading layer," IEEE Electron Device Lett., vol. 34, no. 5, pp. 611613, May 2013.

[25] P. P. Maaskant, H. Shams, M. Akhter, W. Henry, M. J. Kappers, D. Zhu, C. J. Humphreys, and B. Corbett, "High-speed substrateemitting micro-light-emitting diodes for applications requiring high radiance," Appl. Phys. Express, vol. 6, no. 2, p. 022102, Jan. 2013.

[26] J. M. Wun, C. W. Lin, W. Chen, J. K. Sheu, C. L. Lin, Y. L. Li, J. E. Bowers, J.-W. Shi, J. Vinogradov, R. Kruglov, and O. Ziemann, "GaN-based miniaturized cyan light-emitting diodes on a patterned sapphire substrate with improved fiber coupling for very high-speed plastic optical fiber communication," IEEE Photonics J., vol. 4, no. 5, pp. 1520-1529, 2012.

[27] Y. Wang, Y. Xu, T. Yang, X. Gao, B., Zhu, W. Cai, J. Yuan, R. Zhang, H. Zhu, "Simultaneous light emission and detection of InGaN/GaN multiple quantum well diodes for in-plane visible light communication on a chip", Optics Communications. 387, 2016, 10.1016/j.optcom.2016.10.070.

[28] C. Foucher et al., "Flexible Glass Hybridized Colloidal Quantum Dots for $\mathrm{Gb} / \mathrm{s}$ Visible Light Communications", IEEE Photonics Journal, PP (99): 1-1, January 2018, DOI: 10.1109/JPHOT.2018.2792700.

[29] A. Scheianu and G. Suciu, "Hybrid VLC Communication system for Small to Medium Size enterprises", ECAI 2019, June 27-29, to be published in ECAI proceedings.

[30] K. Evans, A. Jones, A. Preece, F. Quevedo, D. Rogers, I. Spasić, I. Taylor et al. "Dynamically reconfigurable workflows for time-critical applications." In Proceedings of the 10th Workshop on Workflows in Support of Large-Scale Science, p. 7. ACM, 2015. 